\title{
Foundational Proof Checkers with Small Witnesses
}

\author{
Dinghao Wu \\ Princeton University \\ \{dinghao, appel\}@cs.princeton.edu
}

Andrew W. Appel

\author{
Aaron Stump \\ Washington University in St. Louis \\ stump@cs. wustl. edu
}

\begin{abstract}
Proof checkers for proof-carrying code (and similar) systems can suffer from two problems: huge proof witnesses and untrustworthy proof rules. No previous design has addressed both of these problems simultaneously. We show the theory, design, and implementation of a proof-checker that permits small proof witnesses and machine-checkable proofs of the soundness of the system.
\end{abstract}

\section{INTRODUCTION}

In a proof-carrying code (PCC) system [10], or in other proof-carrying applications [3], an untrusted prover must convince a trusted checker of the validity of a theorem by sending a proof. Two of the potential problems with this approach are that the proofs might be too large, and that the checker might not be trustworthy. Each of these problems has been solved separately; in this paper we show how to solve them simultaneously.

The general approach is to write a logic program that has a machine-checked semantic correctness proof; this technique can be used in other domains (besides "proof-carrying") to write logic programs with machine-checked guarantees of correctness.

\subsection{Small proof witnesses}

Necula has a series of results on reducing proof size. He represents logics, theorems, and proofs in the notation of the Edinburgh Logical Framework (LF) [8]. But the natural representation of an LF proof contains redundancy (common subexpressions) that can cause exponential blowup if the proofs are written in the usual textual representation. Necula's $\mathrm{LF}_{i}$ [11] data structure eliminated most of this redundancy, leading to reasonable-sized proof terms.

In the PCC framework, given a machine-language program, the proof is of a theorem that the program obeys some safety property. It's natural to compare the size of the representa-

DRAFT OF April 9, 2003 tion of the proof witness to the size of the binary machinelanguage program. Necula's $\mathrm{LF}_{i}$ proof witnesses were about 4 times as big as the programs whose properties they proved.

Pfenning's Elf and Twelf systems [14, 15] are implementations of the Edinburgh Logical Framework. In these systems, proof-search engines can be represented as logic programs, much like (dependently typed, higher-order) Prolog. Elf and Twelf build proof witnesses automatically; if each logic-program clause is viewed as an inference rule, then the proof witness is a trace of the successful goals and subgoals executed by the logic program. That is, the proof witness is a tree whose nodes are the names of clauses and whose subtrees correspond to the subgoals of these clauses.

Necula's theorem provers were written in this style, originally in Elf and later in a logic-programming engine that he built himself. In later work, he moved the prover clauses into the trusted checker. In principle, proof witnesses for such a system can be just a single bit, meaning, "A proof exists: search and ye shall find it." However, to guarantee that proof-search time (in the trusted checker) would be small, Necula invented oracle-based checking [12]: the untrusted prover would record a sequence of bits that recorded which subgoals failed (and therefore, where backtracking was required). This bitstream serves as an "oracle" that the trusted checker can use to avoid backtracking. The oracle bitstream need not be trusted; if it is wrong, then the trusted checker will choose the wrong clauses to satisfy subgoals, and will fail to find a proof.

Using oracle-based checking, the proof witness (the oracle bitstream) is about $1 / 8$ the size of the machine code. ${ }^{1}$ The key idea is to run a simple Prolog engine in the trusted proof checker; the oracle is just an optimization to ensure that the checker doesn't run for too long.

\footnotetext{
${ }^{1}$ Unfortunately, this statistic is somewhat misleading. A "pure" PCC system would transmit two components from an untrusted code producer to a code consumer: a machinelanguage program and a proof witness. The SpecialJ proofcarrying Java system on which Necula measured oraclebased checking transmits three components: The machine code, the proof, and a Java "class file". The Java class file, as is usual in any Java system, contains descriptions of the types of all procedures (methods) in the program, including formal parameter and result types. This information should really be considered part of the proof witness.
} 


\subsection{Trustworthy checkers}

Necula's oracle-based checker for PCC comprises approximately 26,000 lines of code:

\begin{aligned} 23,000 & Verification-condition generator, written in C \\ 1,400 & LF proof checker, written in C \\ 800 & Oracle-based Prolog interpreter, in C \\ 700 & Axioms for type system, written in LF \\ \hline 26,000 & Total trusted lines of code \end{aligned}

The largest component is the verification condition generator (VC-Gen), which traverses the machine-language program and extracts a formula in logic, the verification condition, which is true only if the program obeys a given safety policy.

This 26,000 lines forms the trusted code base (TCB) of the system: any bug in the TCB may cause an unsafe program to be accepted. The large VC-Gen component is a concern, but so are the axioms of the type system: if the type system is not sound, then unsafe programs will be accepted, and League et al. [9] have shown that one of the SpecialJ typing rules is unsound.

The goal of our research [2] has been to check proofs of program safety using a much smaller TCB. We do this by eliminating the VC-Gen component-we reason directly about machine code in higher-order logic, instead of the two-step process of extracting the verification condition and then proving it; and we write the rules of our type system as machine-checkable lemmas, instead of axioms. We have shown that the TCB for a proof-carrying code system can be reduced below 2700 lines, as follows [6]:

803 LF proof checker, written in $\mathrm{C}$

135 Axioms \& definitions of higher-order logic, in LF

160 Axioms \& definitions for arithmetic, in LF

460 Specification of Sparc instruction encodings, in LF

1,005 Specification of Sparc instruction semantics, in LF

105 Specification of safety predicate, in LF

2,668 Total trusted lines of code

Unfortunately, in this prototype system the proof witnesses are huge: the DAG representation of a safety proof of a program might be 1000 times as large as the program. Proof size is approximately linear in the size of the program, ${ }^{2}$ so this factor of 1000 will not grow substantially worse for larger programs. However, while this early prototype is useful in showing how small the TCB can be made, it is impractical for real applications because the proof witnesses are too big.

\subsection{Synthesis}

In this paper we will show that Necula's insight (run a resource-limited Prolog engine in the trusted checker) can be combined with our paranoia (don't trust the logic-programming rules used by such a Prolog engine) to make a PCC checker with small witnesses and a small trusted base.

\footnotetext{
${ }^{2}$ Technically, proof size is expected to be roughly proportional to the size of the program multiplied by the average number of live variables on entry to a basic block; this is superlinear but much less than quadratic, for typical programs.
}

Our approach is as follows. We write a type-checking algorithm in a subset of Prolog with no backtracking, a very limited form of unification, and with efficiently indexed dynamic atomic clauses. We show that the operators of such a Prolog program can be given a semantics in higher-order logic, such that the soundness of each clause can be proved as a machine-checkable lemma. We show that this Prolog subset is adequate for writing efficient type-checkers for PCC and for other "proof-carrying" applications.

Our trusted checker will be sent the Prolog clauses, with machine-checkable soundness proofs; it will check these proofs before installing the clauses. Then it will be sent a theorem to check (i.e., in a PCC application, the safety of a particular machine-language program) and a small proof witness. The Prolog program will traverse the theorem and proof witness; this traversal succeeds only if the theorem is valid.

The TCB of our new checker is only 244 lines larger than our previous prototype. It includes all the components of our previous system (2668 lines) plus a concise implementation of an interpreter for our Prolog subset.

\section{A FOUNDATIONAL TYPE CHECKER}

We will illustrate our approach using an example - a type checker for a very simple programming language. In this example we illustrate the following points, which are common to many proof-carrying applications:

- The specification of the theorem to be proved is quite simple (in this case, that the program evaluates to an even number).

- The proof technique involves the definition of a carefully designed set of predicates that allow a simple, syntax-directed decision procedure (in this case, we define a syntax-directed type system for evenness and oddness).

- The syntax-directed rules are provable, from the definitions of the operators, as machine-checkable lemmas in the underlying higher-order logic (this is what foundational means: the rules are provable from the foundations of logic).

- The syntax-directed rules require management of a symbol table, or context, that would lead to an $O\left(N^{2}\right)$ algorithm if implemented naively; we want a lineartime prover, and we'll show how to make one.

- The language being typechecked in a proof-carrying code system (or in proof-carrying authentication) is the output of another program-the compiler (or a prover). Such languages don't need all of the syntactic sugar that human-readable languages have, and processing them is therefore easier.

\subsection{Example: even-valued expressions}

Consider a simple calculus for expressions with constants, variables, addition, and let-binding, as shown in Figure 1.

A program consists of a list of declarations and an expression. An expression is either a variable, a natural number, 


$$
\begin{array}{llll}
\text { type } & \tau & ::= & \text { even } \mid \text { odd } \\
\text { decl } & d & ::= & \cdot \mid \text { let } x=e ; d \\
\text { expr } & e & ::= & x|n| e_{1}+e_{2} \\
\text { prog } & p & ::= & (d ; e)
\end{array}
$$

Figure 1: Syntax of even-odd system.

$$
\begin{aligned}
& \text { Var } \quad \equiv \text { Num } \\
& \text { State } \quad \equiv \text { Var } \rightarrow \text { Num } \rightarrow \text { Form } \\
& \text { Decl } \equiv \text { State } \rightarrow \text { Form } \\
& \text { Exp } \quad \equiv \text { State } \rightarrow \text { Num } \rightarrow \text { Form } \\
& \text { Program } \equiv\langle\text { Decl, Exp }\rangle \\
& (d ; e) \quad \equiv\langle d, e\rangle \\
& \begin{aligned}
& \equiv \lambda s . \text { true } \\
\text { let } x=e ; d & \equiv \lambda s . d s \wedge(\forall \text { a.e } s a \Rightarrow s x a)
\end{aligned} \\
& x \quad \equiv \lambda s . \lambda a . s x a \\
& n \quad \equiv \lambda s \cdot \lambda a \cdot a=n \\
& e_{1}+e_{2} \equiv \lambda s \cdot \lambda a . \exists a_{1} \cdot \exists a_{2} . \\
& e_{1} s a_{1} \wedge e_{2} s a_{2} \wedge \\
& a=a_{1} \text { plus } a_{2} \\
& \text { safe } \quad \equiv \lambda p . \forall s . f s t(p) s \Rightarrow \\
& \exists a . \operatorname{snd}(p) s a \wedge \text { isEven }(a)
\end{aligned}
$$

Figure 2: Safety specification.

or the sum of two expressions. Here is an example:

$$
\text { let } x=4 \text {; let } y=x+8 ; x+y
$$

There are two declarations followed by an expression; the program evaluates to 12 .

\subsection{Safety specification}

In this simple example, we define that a "safe" program is one that evaluates to an even number. In order to define the safety theorem, we need to know what a program means and how to evaluate a program. The safety theorem, along with a conventional denotational semantics of the language in consideration, is shown in Figure 2.

All of these definitions are treated as axiomatic by our checker; that is, they are trusted. Variables are represented as numbers. A abstract State maps a variable to its content, i.e. a number. A program is a pair of a declaration and an expression; its semantics is the pair of semantics of the corresponding declaration and expression. Declaration Decl is a predicate on states. Expression Exp is a predicate on a state and a number; that is, given a state the expression evaluates to a number. The semantics of concrete expressions is straightforward from definitions.

Finally, the safety theorem is based on the semantics of language constructs. ${ }^{3}$ Given a program $p$, it is "safe" if: for all states $s$, if the declaration of the program, i.e. $f s t(p)$, holds

\footnotetext{
${ }^{3}$ For our PCC application, there are only two language constructs for the machine code to be proved safe. The machine code is a sequence of integers encoding machine instructions; so we only need cons and nil.
}

$$
\begin{aligned}
& \frac{\vdash_{p} p: \text { even }}{\operatorname{safe}(p)} \text { SafeTy } \quad \frac{\cdot \vdash_{d}(d ; e): \tau}{\vdash_{p}(d ; e): \tau} \text { ProgTy } \\
& \frac{\Gamma \vdash_{e} e_{1}: \tau_{1} \quad \Gamma\left[x: \tau_{1}\right] \vdash_{d}(d ; e): \tau}{\Gamma \vdash_{d}\left(\operatorname{let} x=e_{1} ; d ; e\right): \tau} \text { DeclConsTy } \\
& \frac{\Gamma \vdash_{e} e: \tau}{\Gamma \vdash_{d}(\cdot ; e): \tau} \text { DeclNilTy } \quad \frac{\Gamma(x)=\tau}{\Gamma \vdash_{e} x: \tau} \operatorname{VarTy} \\
& \frac{\Gamma \vdash_{e} e_{1}: \tau_{1} \quad \Gamma \vdash_{e} e_{2}: \tau_{2} \quad \tau_{1} \boxplus \tau_{2}=\tau}{\Gamma \vdash_{e} e_{1}+e_{2}: \tau} \text { PlusTy } \\
& \overline{\text { even } \boxplus \text { even }=\text { even }} \boxplus e e \quad \overline{\text { odd } \boxplus \text { odd }=\text { even }} \boxplus \circ o \\
& \overline{\text { even } \boxplus \text { odd }=\text { odd }} \boxplus e o \quad \overline{\text { odd } \boxplus \text { even }=\text { odd }} \boxplus o e
\end{aligned}
$$

Figure 3: Typing rules with static context.

on $s$, then there exists a number $a$ such that the expression of the program, i.e. $\operatorname{snd}(p)$, evaluate to $a$ and $a$ is even.

\subsection{Type checker}

The typing rules appear in Figure 3. There are three kinds of typing judgements. The judgement for a program $\vdash_{p}$ checks that the program evaluates to a number whose type is $\tau$. The declaration judgement $\vdash_{d}$ states that, assuming the environment built so far, and assuming the remaining declarations hold, the expression has a certain type. The expression judgement $\vdash_{e}$ asserts that an expression has certain type under typing context $\Gamma$.

These typing rules can be read as a Prolog-like logic program. Each rule is a clause of the logic program. The conclusion of a rule is the head of the clause, and each premise of the rule is a subgoal. The typing rules are designed such that the conclusions of these typing rules are disjoint. Therefore, when running the type checker (as a logic program) there is no need to backtrack; we say that such a type system is syntax-directed.

Furthermore, if we give denotational semantics based on higher-order logic to typing judgements such as $\vdash_{p}, \vdash_{d}$, and $\vdash_{e}$, each typing rule can be proved as a lemma in the system, thus its soundness is guaranteed with respect to the foundations of logic. The denotational sematics of typing judgements is given in Figure 4. Proofs of the typing rules are quite straightforward and thus omitted here. The denotational semantics of the type operators are part of the safety proof, not part of the safety specification. That is, they are not trusted. It is straightforward to prove the safety theorem from the conclusion of type checking rule ProgTy if we pass $\tau$ even when invoking the type checker, as shown in the Safe Ty rule.

Our checker will determine the validity of the safety predicate by determining whether a proof exists. It will not exactly construct such a proof as a data structure: instead, it will traverse a trace of such a proof, composing lemmas in a syntax-directed way. We call our set of lemmas a type system: our machine-checked safety proof of a program $P$ 


$$
\begin{array}{ll}
\text { Ty } & \equiv \text { Num } \rightarrow \text { Form } \\
\text { Env } & \equiv \text { State } \rightarrow \text { Form } \\
\text { even } & \equiv \lambda x . \exists n . \text { isInt }(n) \wedge x=2 n \\
\text { odd } & \equiv \lambda x . \exists n . \text { isInt }(n) \wedge x=2 n+1 \\
& \\
\vdash_{p} p: \tau & \equiv f s t(p) s \Rightarrow \exists a . \operatorname{snd}(p) s a \wedge \tau a \\
\Gamma \vdash_{d}(d ; e): \tau & \equiv \forall s .(d s \wedge \Gamma s) \Rightarrow \exists a .(e s a \wedge \tau a) \\
\Gamma \vdash_{e} e: \tau & \equiv \forall s . \Gamma s \Rightarrow \exists a .(e s a \wedge \tau a) \\
\tau_{1} \boxplus \tau_{2}=\tau & \equiv \forall n_{1} . \forall n_{2} \cdot \tau_{1} n_{1} \Rightarrow \tau_{2} n_{2} \Rightarrow \tau\left(n_{1}+n_{2}\right) \\
\Gamma[x: \tau] & \equiv \lambda s . \Gamma s \wedge \exists a . s x \wedge \tau a \\
\Gamma(x)=\tau & \equiv \forall s . \Gamma s \Rightarrow \exists a . s x a \wedge \tau a
\end{array}
$$

Figure 4: Definitions of types and judgements.

consists of (1) a proof of soundness for the type system, and (2) the successful syntax-directed execution of the typing clauses as applied to $P$.

Efficiency and proof size problem. When type checking a program, we build a type environment, or context, from the declarations for variables that appear in the expression. The rules for traversing a list of declarations and building the corresponding type contexts are DeclConsTy and DeclNilTy. When a variable is encountered, we look up its type in the context. However, the typing rule VarType does not specify a context lookup algorithm. Consider the following variable type-lookup rules.

$$
\begin{gathered}
\overline{\Gamma[x: \tau] \vdash x: \tau} \text { VarTyHit } \\
\frac{\Gamma \vdash x: \tau \quad x \neq y}{\Gamma\left[y: \tau^{\prime}\right] \vdash x: \tau} \text { VarTyMiss }
\end{gathered}
$$

Suppose the context is simply organized as a list in these two rules; each element of the list is a pair: a variable and its type. Then each context lookup takes linear time, and typechecking a whole program will take quadratic time. Furthermore, the size of the generated proof for a lookup operation is linear with respect to the size of the context, and thus the safety proof for a program has a quadratic blowup. In the next section, we give a more efficient algorithm that still has a provably sound semantic model, and generates concise proofs.

\section{EFFECTIVE CONTEXT MANAGEMENT}

As we have explained, we avoid sending (very large) proofs to the trusted checker by sending a proof scheme (with a soundness proof for the proof scheme). We want the proof scheme to "execute" efficiently, that is, in linear time with respect to the size of the program-safety-theorem being proved. And we want the proof schemes to be written in the "smallest possible" Prolog-like language: what set of language features are useful?

Here we will show an efficient proof scheme for contexts; because this scheme requires dynamic clauses in the Prolog subset, we have included a limited form of dynamic clause in our language design.

\subsection{Dynamic clauses and local assumptions}

Many logic programming systems provide a facility for managing dynamic clauses at run time. In Prolog, users can assert a fact or clause into database or retract back a clause dynamically. The assert/retract mechanism can be expensive if the dynamic clause in consideration is not atomic (i.e., has subgoals) because the dynamic clause has to be compiled and integrated into the program's decision trees. If the dynamic clause is atomic, with input-mode arguments that are integers or hashable, the assert/retract operation can be cheap: Prolog systems usually provide efficient support for asserting or retracting an atomic clause by using hash tables. That is, asserting, retracting, and querying indexable atomic clauses can be done in constant time per operation.

In Logical Framework (LF) [8], or its implementaion Twelf $[13,15]$, one can use local assumptions [16] to check dynamic clauses into database. Since these assumptions are local, their static scopes control their lifetimes; there is no need to provide an explicit retract mechanism. A clause of the form

$$
\{x: \tau\} A x \rightarrow B x
$$

introduces a local assumption $A x$ into the context and then solves the goal $B x$ under this assumption. ${ }^{4}$ When proof search on goal $B$ has finished, assumption $A$ is automatically retracted. That is, Twelf uses a dynamically well-scoped version of assert/retract. One can use Prolog assert/retract mechanism to simulate Twelf's local assumptions, however. We can give semantics to local assumptions and generate concise proofs so that clauses are guaranteed to be correct.

Local assumptions are particularly effective-efficient, secure (with a provably sound model), and concise - when we need to deal with big environemnts and generate proofs of lookups in the environment as well.

\subsection{Typing rules}

In this subsection, we present an efficient type checking algorithm using dynamic clauses. We give semantics in the next subsection. Figure 5 shows typing rules with a dynamic context management scheme.

The rule ProgTy calls a declaration checking rule and passes declaration $d$ to it. The declaration $d$ appears twice in the premise: The declaration checking rules traverses one $d$, and the other $d$ is used to pass the original declatation all the way to the expression checking rules.

The rule BindTy is worth some explanation. Informally, it scans the list of declarations, checks them in dynamically, and continues type checking. It first checks that the expression $e_{1}$ has type $\tau_{1}$, then asserts this fact as a dynamic clause (or local assumption) $\operatorname{bind}\left(x, \tau_{1}, \Gamma\right)$ and continues type checking.

When type checking a variable expression, the rule VarTy tries to match the previous checked-in local assumptions. The lookup operation takes constant time and the proof generated for it is concise. The $\boxplus$ rules remain the same as before.

\footnotetext{
${ }^{4}$ It is a dependent type on local parameter $x$.
} 


$$
\begin{aligned}
& \frac{\vdash_{p} p: \text { even }}{\operatorname{safe}(p)} \text { SafeTy } \quad \frac{d \vdash_{d}(d ; e): \tau}{\vdash_{p}(d ; e): \tau} \text { ProgTy } \\
& \frac{\Gamma \vdash_{e} e_{1}: \tau_{1} \quad \operatorname{bind}\left(x, \tau_{1}, \Gamma\right) \rightarrow \Gamma \vdash_{d}(d ; e): \tau}{\Gamma \vdash_{d}\left(\text { let } x=e_{1} ; d ; e\right): \tau} \operatorname{BindTy} \\
& \frac{\Gamma \vdash_{e} e: \tau}{\Gamma \vdash_{d}(\cdot ; e): \tau} \operatorname{BindNil} \quad \frac{\operatorname{bind}\left(x, \tau_{1}, \Gamma\right)}{\Gamma \vdash_{e} x: \tau} \operatorname{VarTy} \\
& \frac{\Gamma \vdash_{e} e_{1}: \tau_{1} \quad \Gamma \vdash_{e} e_{2}: \tau_{2} \quad \tau_{1} \boxplus \tau_{2}=\tau}{\Gamma \vdash_{e} e_{1}+e_{2}: \tau} \text { PlusTy } \\
& \overline{\text { even } \boxplus \text { even }=\text { even }} \boxplus e e \quad \overline{\text { odd } \boxplus \text { odd }=\text { even }} \boxplus o o \\
& \overline{\text { even } \boxplus \text { odd }=\text { odd }} \boxplus e o \quad \overline{\text { odd } \boxplus \text { even }=\text { odd }} \boxplus o e
\end{aligned}
$$

Figure 5: Typing rules with dynamic context.

In a conventional Prolog implementation that supports efficient assert/retract operations for atomic dynamic clauses like $\operatorname{bind}\left(x, \tau_{1}, \Gamma\right)$, the type checking algorithm above is linear. Moreover, it is provably sound as shown in the next subsection.

\subsection{Foundational semantics and proofs}

The safety specification remains the same as presented in Figure 2. The definitions of types and typing judgements remain untouched except for $\vdash_{d}$ and the new constructor bind.

$$
\begin{array}{rc}
\Gamma \vdash_{d}(d ; e): \tau \equiv & \forall s .(\Gamma \sqsubseteq d \wedge \Gamma s) \Rightarrow \\
\operatorname{bind}(x, \tau, \Gamma) \equiv & \exists a \cdot(e s a \wedge \tau a) \\
& \forall s . \Gamma s \Rightarrow \\
d_{1} \sqsubseteq d_{2} & \exists a \cdot(s x a \wedge \tau a) \\
& \forall s . d_{1} s \Rightarrow d_{2} s
\end{array}
$$

The semantics of dynamic clause $\operatorname{bind}(x, \tau, \Gamma)$ is very similar to that of the static binding operator $\Gamma[x: \tau]$ and lookup operator $\Gamma(x)=\tau$. It serves both purposes. From these definitions it is straightforward to prove the typing rules as lemmas and the safety theorem can be proved from the successful type checking of a program from the goal $\vdash_{p}(d ; e)$ : even. Here we give the proof for rule BindTy.

$$
\begin{aligned}
& \text { LEMma } 1 \quad(\text { BindTy). } \\
& \qquad \frac{\Gamma \vdash_{e} e_{1}: \tau_{1} \quad \operatorname{bind}\left(x, \tau_{1}, \Gamma\right) \rightarrow \Gamma \vdash_{d}(d ; e): \tau}{\Gamma \vdash_{d}\left(\text { let } x=e_{1} ; d ; e\right): \tau}
\end{aligned}
$$

Proof. By definition of $\vdash_{d}$, for all state $s$, we assume $\Gamma \sqsubseteq$ (let $\left.x=e_{1} ; d\right)$ and $\Gamma s$, then we prove $\exists a$. $($ e $s a \wedge \tau a)$. This can be obtained from $\Gamma \vdash_{d}(d ; e): \tau$. In order to use this fact, we need to prove the local assumption $\operatorname{bind}\left(x, \tau_{1}, \Gamma\right)$, which can be proved from the premise $\Gamma \vdash_{e} e_{1}: \tau_{1}$ and the assumption $\Gamma \sqsubseteq\left(\right.$ let $\left.x=e_{1} ; d\right)$.
The machine checkable proof for this rule can be found in Appendix A.

\section{FLIT}

The previous section illustrated that efficient syntax-directed type-checking uses certain logic-programming constructs (dynamic clauses) but not others (backtracking), and that each Horn clause can be proved sound as a lemma in higherorder logic. This section describes a suitable logic programming interpreter implemented in Flit, our trusted LF proof checker. Other aspects of Flit are described in another paper [6]. To achieve a concise and efficient implementation, we impose several restrictions on the form of goals and programs. If these are violated, the interpreter will remain sound but may fail to be complete. This section discusses these restrictions and the implementation of the interpreter. We begin with a few basic definitions from logic programming.

\subsection{Basic definitions}

Flit's logic programming interpreter can solve goals with respect to logic programs containing dynamic clauses and simple arithmetic. Goals are atomic formulas (also called atoms) of first-order logic. Logic programs are conjunctions of clauses, where each clause is either a universally quantified atom or else a universally quantified implicational formula formed from atoms using only conjunctions and implications. Implications other than the topmost one must be in the antecedents of other implications. Nested implications give rise to dynamic clauses. As usual, the head of a clause is the clause itself for atomic clauses and the consequent of the topmost implication for implicational clauses. The body of a clause is its antecedent if it is an implicational clause, and TRUE if it is an atomic clause. We assume the usual notion of unification of atoms, based on the usual notion of substitution for a finite set of variables. A substitution is ground if all terms in its range are ground. Goals and subgoals are just atoms. A solution for a goal $G$ with respect to a logic program $\mathcal{P}$ is a substitution $\sigma$ such that $(\mathcal{A} \cup \mathcal{P}) \vdash \sigma(G)$, where $\vdash$ is provability in first-order logic and $\mathcal{A}$ are axioms for addition, multiplication, and truncating division on 32-bit natural numbers. These arithmetic operations are represented as three-place relations, where the last place gives the output of the operation. ${ }^{5}$

For reasoning about our logic programming interpreter, we will make use of a standard natural deduction proof system for first-order logic. Then a use of a clause $C$ in a proof is either an assumption of $C$, if $C$ is atomic; or an application of modus ponens whose implicational argument is $C$. The subgoals produced while solving a goal $G$ are just the atomic formulas contained in the proof of $G$ from $\mathcal{A} \cup \mathcal{P}$.

\subsection{Flit's logic programming language}

Flit's logic programming interpreter is sound for arbitrary logic programs with dynamic clauses. It is complete for

\footnotetext{
${ }^{5}$ Since the 32-bit natural numbers are not closed under addition or multiplication, goals such as multiply $2^{20} 2^{20} X$ are not satisfiable. However, our underlying higher-order logic has the complete theory of the integers; the 32-bit numbers are just a way of writing some of the constants and evaluating some of the expressions in that theory.
} 
solving goals $G$ with respect to logic programs $\mathcal{P}$, under certain conditions. The first two of these are not obviously statically checkable, while the rest are statically checkable.

1. Deterministic program. The program $\mathcal{P}$ must be deterministic for $G$, in the following sense. Suppose $G$ is provable from $\mathcal{P}$. Then $G$ has a proof where every subgoal $S$ is proved by a use of the first clause of $\mathcal{D} \cup \mathcal{P}$ whose head unifies with $S$, where $\mathcal{D}$ are the active assumptions (corresponding to active dynamic clauses). Under this condition of determinism, the interpreter need not backtrack to be complete. It is never necessary to try later clauses if a proof cannot be found from a use of the first unifying clause.

2. Bounded execution. $\mathcal{P}$ must be bounded for $G$. This means that if $G$ is provable from $\mathcal{P}$, then there exists a proof whose size is no more than some fixed constant $M A X_{-} P R O O F$. This assumption is used to avoid dynamic allocation of memory while trying to solve $G$.

3. Argument modes. Some of the restrictions needed for the completeness of the interpreter are most easily stated in terms of correct modings. A correct moding indicates how ground terms and numeric constants move through the clauses of the program as inputs, outputs, and indices which will be used for efficient lookup of dynamic clauses. We define a moding of a logic program to be a function assigning to each argument position of each predicate of the program a mode, which is either input, index, or output; and assigning to some argument positions of function symbols the mode index. The arithmetic operations complicate the definition of correct moding, so we give the definition first for programs not using the arithmetic operations.

A moding is correct for a program without arithmetic iff for each clause $C$ of the program with head $H$ and body $B$, the following is true. Let $\sigma_{1}$ be any ground substitution for just the variables in $H$ such that $\sigma_{1}(H)$ contains only ground terms in input position and distinct numeric constants in index positions. Let $\sigma_{2}$ be any ground substitution for just the variables in output positions in $B$. Let $C^{\prime}$ be $\sigma_{1}\left(\sigma_{2}(C)\right)$, and let $B^{\prime}$ be the body of $C^{\prime}$. Then all input positions and output positions of $C^{\prime}$ contain only ground terms, and the index positions of $B^{\prime}$ contain distinct numeric constants, where these are the same numeric constants that occur in $\sigma_{1}(H)$.

For programs with arithmetic, the definition of correct moding is similar, except for how addition is handled. Instead of computing with primitive addition and subtraction operators, we use the addition operator for both: the goal add $34 X$ finds the sum of 3 and 4 , while add $3 X 7$ finds the difference between 7 and 3. Unfortunately, this means that addition does not have a simple, global moding. We require instead that for any substitution $\sigma_{1}$ as above, there exists an assignment of modes to the argument positions of the different occurrences of addition in the body $B$ of the clause. For each occurrence, at most one argument position is assigned the mode output and the others are assigned mode input. This now ensures that every argument position of $C$ is moded, without requiring a static moding of addition. We then require that for any $\sigma_{2}$ as above, all the same conditions on $\sigma_{1}\left(\sigma_{2}(C)\right)$ as stated above hold.
Note that our requirement of correct moding ensures that all index positions in the body of a clause are either numeric constants or variables which occur in index positions in the head of the clause. In particular, we disallow using the output of a subgoal, particularly an arithmetic subgoal, as an index. A simple inductive argument then shows that all numeric constants used as indices in subgoals while solving a goal must occur in index positions of that goal.

Correctness of a moding can be statically checked with a simple linear-time algorithm. Flit does not check modings statically, however, because incorrect moding will lead only to incompleteness, not unsoundness.

\section{Dynamic clauses must be atomic.}

5. Output arguments. All output positions in the goal and the program must contain either a ground term or a variable. If an output position of an atom $A$ contains a variable $v$, then $v$ is called an output variable of $A$.

6. No repeated output variables. No output variable can be used twice in the goal or in the same atom of the program.

Prolog interpreters typically enter atomic dynamic clauses in hash table for efficient matching, using one of the predicate's arguments as the hash key. Our logic programs can be written with a very restricted form of clause indexing:

7. Index position. The first argument position of any predicate used in a dynamic clause must be its only index position.

8. Bounded indexes. The numeric constants appearing in index positions must be smaller than some fixed constant. $M A X_{-} I N D E X$.

Example. The even-odd proof scheme of Figure 5 is a logic program that conforms to these restrictions. The proof scheme is (1) syntax-directed, (2) executes in linear time and space, (3) is well moded, (4) the dynamic clauses $\operatorname{bind}(x, \tau, \Gamma)$ are all atomic, and the output arguments of predicates conform to rules (5) and (6). In our implementation of this proof scheme, we put the $x$ argument of $\operatorname{bind}(x, \tau, \Gamma)$ in the first position to conform to the (7) index position rule; and (8) all the indices $x$ are manifest constants that are small integers.

Our LTAL proof scheme used in the real PCC system also obeys all these restrictions.

\subsection{Constructing programs}

A logic program is presented to Flit's interpreter as a set of LF terms, represented using an expression data structure (expr). These terms are built from the LF constants declared in the trusted computing base. They represent proofs of theorems derivable from the axioms represented by the constants. The type of each term represents the lemma that it proves. The set of these lemmas is essentially the logic program to use. To massage the LF type into a form that is convenient for logic programming, however, the following transformations are applied in order: 
Strip_pi. The LF type is of the form

$$
\Pi x_{1}: \tau_{1} \ldots \Pi x_{n}: \tau_{n} . \Pi a_{1}: G_{1} \ldots \Pi a_{n}: G_{n} . G
$$

where $x_{1}, \ldots, x_{n}$ are the typed logic variables, $G_{1}, \ldots, G_{n}$ are the subgoals of the clause, and $G$ is the head of the clause. Flit distinguishes $\Pi$-abstractions declaring logic variables and $\Pi$-abstractions stating subgoals by checking whether the type of a $\Pi$-abstraction is an application of a logic programming predicate or not. The interpreter is told which are the predicates by marking the exprs with a flag. The type is then transformed to

$$
\Pi a_{1}: G_{1} \ldots \Pi a_{n}: G_{n} \cdot G
$$

The logic variables $x_{1}, \ldots, x_{n}$ are marked with a different flag, for use in subsequent processing.

Massage_type. As it stands so far, the head of the clause is buried beneath $\Pi$-abstractions stating the subgoals of the clause. For more efficient matching, we next massage the type to get the following:

$$
G \leftarrow\left(G_{n},\left(\ldots,\left(G_{2}, G_{1}\right)\right)\right)
$$

This puts the head at the top of the expr, and ensures that subgoals will be solved in the appropriate order, with $G_{n}$ solved first.

Rename_vars. Finally, all the logic programming variables of the clause are replaced by distinct fresh variables.

This variable renaming establishes the following property, which is an invariant of the interpreter for all logic programs and goals that satisfy the requirements of Section 4.2:

INVARIANT 1 . The set of logic variables of any goal is disjoint from the set of logic variables of the logic program.

This invariant is needed for completeness, not soundness, of the interpreter. How it is used is explained in Section 4.5 below.

Finally, for a clause to add an atomic dynamic clause when checking a particular subgoal $G$, it should contain an expression of the form $\Pi a: G . G^{\prime}$ in its list of subgoals.

\subsection{Solving goals}

After a logic program has been obtained in the way described in the previous Section, Flit's logic programming interpreter can solve goals with respect to that program. The algorithm to do this is given in pseudocode in Figure 6. This run_lp() function returns a boolean indicating whether or not a solution was found for the goal. It always terminates, even if one of the conditions described in Section 4.2 is violated. In the latter case, the function may abort where a more powerful interpreter would succeed. If a solution is found, a single global substitution is updated to hold the unifier.

There are three cases in the code of Figure 6. The first is for solving a pair of subgoals. This is done by first solving the first. If this succeeds, then the global unifier has been updated with the solution to the goal. This unifier is applied to the second subgoal, and the result is solved. For efficiency, applying the global unifier to an expression always renames the logic variables of that expression which are not being mapped by the unifier. This ensures that logic variables in subgoals are always different from the logic variables in the program. This helps preserve Invariant 1.

The second case of run_lp() is for dynamic clauses. We add the dynamic clause $\mathrm{G}$, then solve the subgoal G', and then remove the dynamic clause. The restrictions on programs in Section 4.2 ensure that the index position of the dynamic clause, which is its first argument position, will always be a numeric constant $n$ less than some fixed compile-time bound at this point. So we just update a global array, which is declared to be of size equal to this compile-time bound, at index $n$ to hold the dynamic clause. The restrictions on programs also ensure that no dynamic clause with the same index $n$ will be added by the call run_lp (G') which is made while this new dynamic clause is active. So there is no need to worry about collisions in our array of dynamic clauses.

The default case of run_lp() is for atomic goals. This is the only other form of goal allowed by the conditions of Section 4.2. To solve an atomic goal, we try to find a clause whose head unifies with the goal. If this fails, run_lp() aborts, indicating that the goal cannot be solved. Otherwise, it either succeeds if the clause is atomic, or else applies the unifier to the clause's subgoals and tries to solve them by recursive call to run_lp(). If a clause has more than one subgoal, they are already packaged up in order using commas, so the first case of run_lp() will be used in the recursive call to solve them in order.

Finally, statements at the beginning and end of run_lp() are used to recycle the memory for all the expressions generated during the execution of run_lp(). This is done just by resetting the index of the next expression node to take out of the global fixed-size array of expression nodes when creating a new expression. Recycling all those expressions seems to be important for keeping memory usage reasonably low. The condition of boundedness in Section 4.2 above ensures that if a goal is solvable, it has a proof whose size $s$ is less than some fixed constant MAX_PROOF. By recycling expressions after each recursive call to $\operatorname{run} \_p()$, we guarantee that run_lp() can actually find a proof using memory proportional to the longest path through $s$, rather than $s$ itself. The next section discusses an implementation trick needed for soundness of this optimization.

\subsection{Unification}

The top-level routine for solving a goal given in Figure 6 relies on a subroutine to find a clause whose head unifies with an atomic goal. This subroutine works as follows. First, it checks if goal is an arithmetic goal. If so, it tries to solve for a single variable, following the moding constraints described in Section 4.2 above. Note that arithmetic goals where overflow or division by zero occurs are considered to fail. If the goal is not an arithmetic one, the subroutine next checks if the first argument of the goal is a numeric constant $n$. If so, it first tries to unify the goal with the dynamic clause, if there is one, in the global array of dynamic clauses at index $n$. If there is no unifying clause there, then the subroutine tries to unify the goal with the head of each clause of the program in order. If it cannot find a unifying clause, it returns null. 


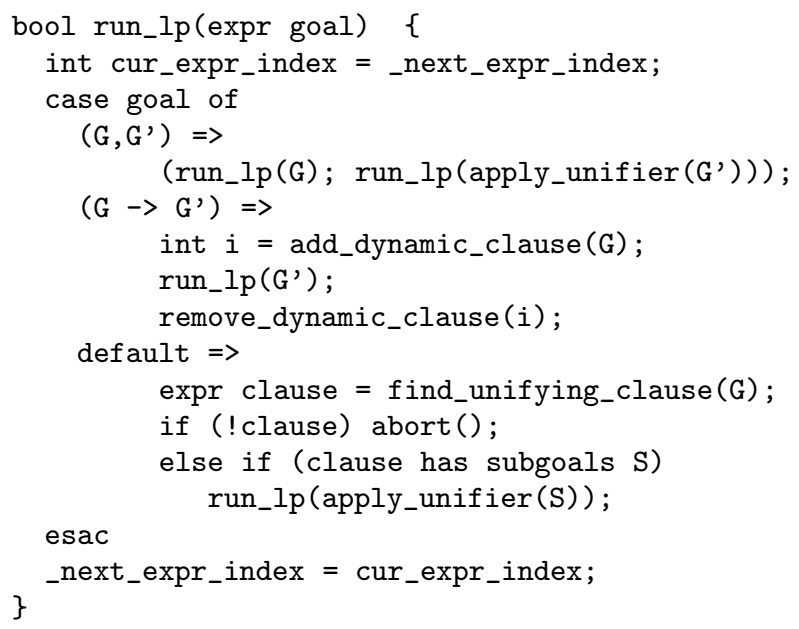

Figure 6: Logic program interpreter

Otherwise, it returns the clause.

Figure 7 gives pseudocode for unification algorithm used by the interpreter. A helper function add_to_unifier_if () is defined in Figure 8, which changes the global substitution to map the pattern to the target and returns true, iff the pattern is a variable not already mapped in the global substitution to some different expression. A single global unifier is implemented by using a field subst of the expr data structure: e.subst points to expression $E$ iff $e$ is a variable mapped to $E$ by the unifier.

To check whether or not a goal unifies with the head of a clause, we call unif() with the goal given as the target and the head of the clause as the pattern. The algorithm proceeds much like a standard syntactic matching algorithm, except for the case where the target is a variable. Under the requirements of Section 4.2, the target must either be ground or a variable. If it is a variable and the pattern is not, then the then-part of the first if statement of unif() will be executed. The second call to add_to_unifier() will then succeed, as long as target was not already mapped to another expression. The latter case can only happen if the same output variable occurs twice in an atom in the goal or the program, which violates a requirement of Section 4.2. The variable will be placed on a list of output variables. Something similar happens in the case where the target and pattern are both variables. If the pattern is already mapped by the substitution to some expression $e$, then the target is also mapped to $e$, and stored in the list of output variables. If the pattern and target are both variables but the pattern is not mapped by the substitution, then we map the pattern to the target. This means that output variables of the goal will be pushed into the subgoals of the clause when the unifier is applied. This enables run_lp() to recycle all expressions generated while solving subgoal $G$, without needing to account for the case where some of those are output variables that are mapped to by an output variable of an earlier subgoal $G^{\prime}$.

We clear the bindings in the global unifier for the set of output variables before trying to match a goal against the head of a new clause. This is the purpose of maintaining the list output_vars. Since no two clauses of the logic program

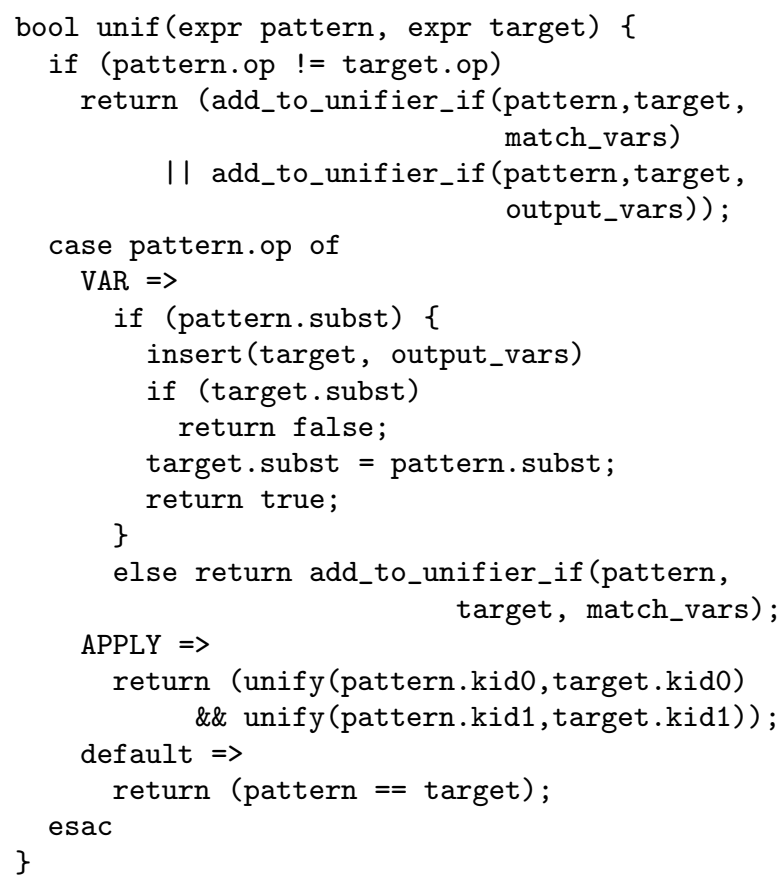

Figure 7: The unification algorithm

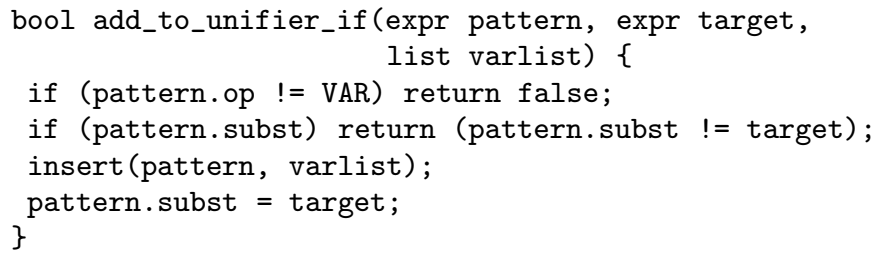

Figure 8: A helper function for unification

contain the same logic variable by Invariant 1, we need to clear the variables from the program just before trying to find a matching clause for a new goal. We keep track of the variables from program clauses in the list match_vars.

\section{PROOF WITNESSES}

Our even-odd example is overly simplistic in that there is a syntax-directed decision procedure for the main safety theorem: for an expression $E$, if the formula $\operatorname{safe}(E)$ is true, then the proof is easily found. In a real proof-carrying code application, the program $e$ is in machine language; loops and recursion in the program, and quantified types in the type system, make type inference impossible.

Thus, in a PCC application, the input to the prover includes the program $E$ and also an untrusted hint $H$. The hint provides loop invariants, type annotations, and other information which can be used by the prover. Because the hint is provided by the same adversary who provides the program, $H$ cannot be assumed accurate, but it can still be useful in constructing the proof.

We will illustrate using the even-odd example. Let us provide a hint $H$ which is a list of type annotations, $x_{1}: \tau_{1}, x_{2}$ : $\tau_{2}, \ldots, x_{n}: \tau_{n}$. We will write a prover that uses this hint (even though for this simple language the hint is not necessary). The root goal is now $\vdash_{p} H E$ instead of $\operatorname{safe}(E)$. 
In addition to running the logic program on the root query $\vdash_{p} H E$, the checker verifies a (static) proof of the lemma,

$$
\frac{\vdash_{p} H E}{\operatorname{safe}(E)} .
$$

We can't use this as a logic-programming rule, i.e. we can't use $\operatorname{safe}(E)$ as our query, because then the logic program would have to "guess" $H$, which could require unbounded backtracking.

The hint $H$ serves as a proof witness for $E$, in conjunction with the Prolog program (i.e. proof scheme) and its semantic soundness proof.

\subsection{Layers of specification and proof}

To handle proof-checking with hints, the checker software must process separately several layers of specification, semantics, proof, and logic-programming clauses. It is useful to think in terms of a proof consumer and an adversary.

\begin{tabular}{r|c|c} 
Trusted $\uparrow$ & Axioms & \multirow{2}{*}{ stage 1 } \\
\cline { 2 - 2 } & Expression Operators & \\
\hline Untrusted $\downarrow$ & Stage 2 \\
& Momantic & \\
\cline { 2 - 2 } Proof scheme & Hint Operators & \multirow{2}{*}{ stage 3 } \\
\cline { 2 - 2 } Theorem to be proved & Clauses & Expression \\
\cline { 2 - 2 } Proof witness & Hint &
\end{tabular}

Stage 1. The proof consumer specifies the Axioms of a logic, and defines the kinds of theorems she wants to checkthat is, the language of expressions for which she wants safety theorems - by defining Expression Operators. One of the expression operators must be a predicate called safe.

Stage 2. Then the adversary sends a proof scheme, that is, a logic program (the syntactic type checker in the even-odd example). This program manipulates goals expressed using the Expression Operators and the Hint Operators. All the hint operators must be defined in terms of the underlying logic - the adversary is not permitted to add uninterpreted operators to the logic. All the Clauses of the logic program must be proved as derived lemmas in the logic, from the definitions of the expression and hint operators, as Lemma 1 does.

The Semantic Model, sent by the adversary, is simply a set of supporting definitions and lemmas, defined in terms of the underlying logic, that can be useful in defining the hint operators and the clauses.

The adversary may define as many hint operators and clauses as he likes; however, there must be one operator called $\vdash_{p}$, and the semantic model must contain a lemma of the form,

$$
\frac{\vdash_{p} H E}{\operatorname{safe}(E)} \text {. }
$$

The proof consumer uses the logical framework (LF) to check the wellformedness of all the definitions and the proofs of all the lemmas. Then she loads the Clauses into the subset-Prolog interpreter.

Stage 3. Finally, the adversary sends an Expression and

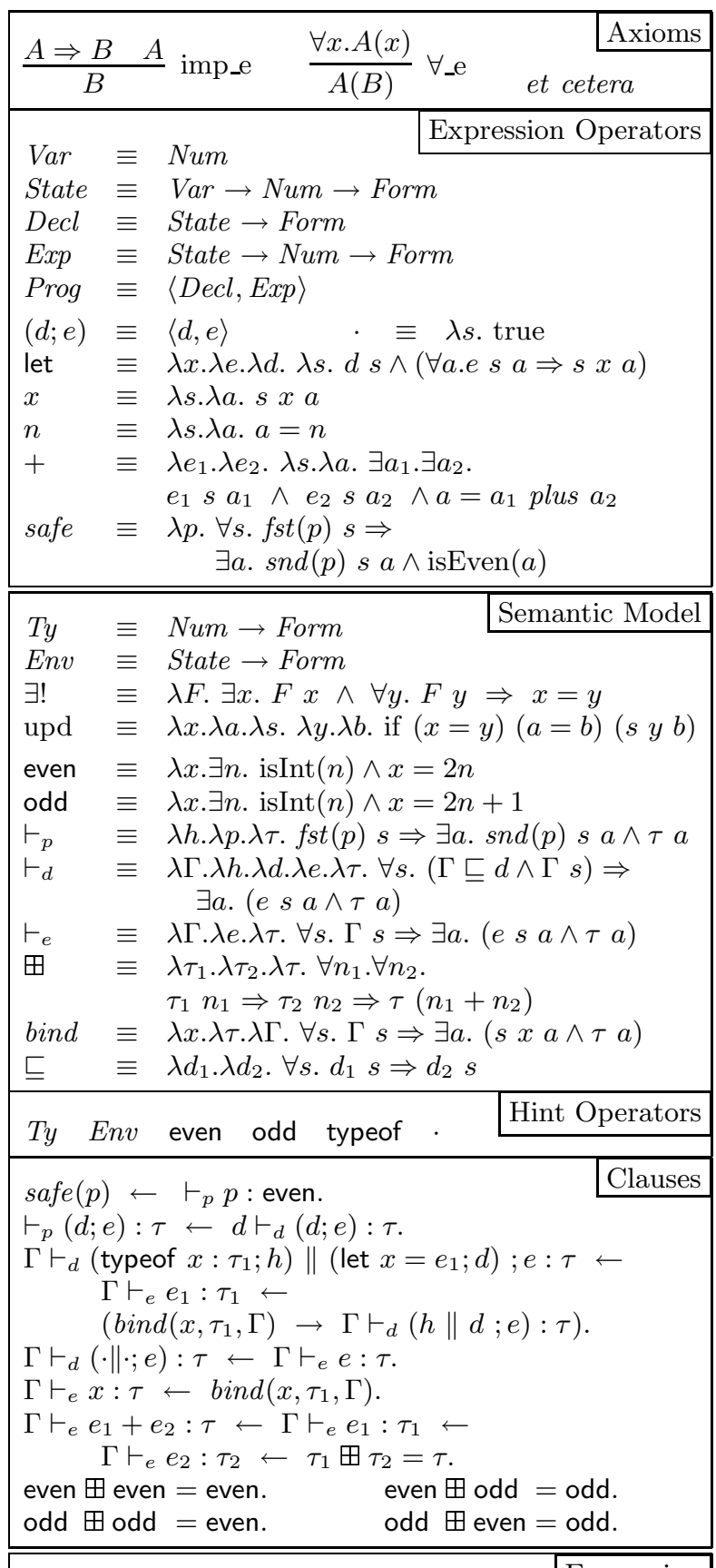

\begin{tabular}{|l|l|}
\hline \hline let $x=4 ;$ let $y=x+8 ; x+y$ & Expression \\
\hline typeof $x$ even (typeof $y$ even $\cdot$ ) & \\
\hline
\end{tabular}

Figure 9: Proof scheme for even-odd system. Not shown are the proofs (in higher-order logic) of all the clauses.

a Hint. The consumer needs to verify that the expression obeys her desired safety property - this was the point of the whole exercise! - and she will do it using the adversary's proof scheme. Since the proof scheme was proved sound (and she has checked the proof), then if the logic program completes successfully, then safe $(E)$ must be valid. 


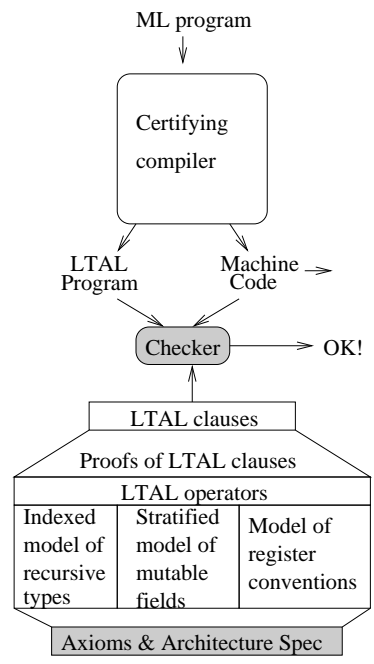

Figure 10: Foundational PCC Framework. Trusted components are shaded.

For the even-odd system, the implementation of these stages is shown in Figure 9; sample source code written in Twelf is in Appendix A.

What is a proof witness? Stage 1 (loading axioms and safety predicate) needs to be done only once per safety policy. In a PCC application, stage 2 (loading the proof scheme) would need to be done when there are substantial modifications to the the untrusted compiler. Stage 3 is repeated for each compiled program sent from the compiler to the consumer. Clearly, any work done in stages 1 and 2 can be amortized over many executions of stage 3. Although the foundational proof derives from information transmitted in stages 2 and 3, in measuring the effective size of proof witnesses we can consider just the Hint sent in stage 3 .

\section{APPLICATION: FOUNDATIONAL PCC}

The even-odd type system is just a toy example to demonstrate some of the principles. Our real applications are in proof-carrying code and distributed authorization. Our checking system scales up to these examples quite well, as we will explain.

In our application to foundational PCC, the hint $H$ is an expression in a calculus called LTAL [7], and the expression $E$ is a machine-language program, that is, a sequence of 32-bit natural numbers.

Figure 10 shows the major components of our foundational proof-carrying code framework. The LTAL clauses are a set of clauses in our restricted Prolog subset. Axioms \& Architecture Spec comprise our Trusted Computing Base (TCB), and are preloaded into our Checker. Between these two components are proofs, based on the axioms, of all the $L T A L$ clauses.

A source program is compiled into a machine-code program and a Low-level Typed Assembly Language (LTAL) expression. The compiler is not trusted, because it is a large program that may have bugs. The trusted checker receives the

\begin{tabular}{|c|c|c|c|}
\hline programs & $P$ & $::=$ & $\left(M, \vec{B}, l_{s}\right)$ \\
\hline basic blocks & $B$ & $::=$ & $f[\vec{\alpha}]\left(v_{1}: \tau_{1}, \ldots, v_{n}: \tau_{n}\right) S$ \\
\hline maps & $M$ & $::=$ & $(L, R, T)$ \\
\hline label map & $L$ & $::=$ & $\left\{l_{1} \mapsto a_{1}, \ldots, l_{n} \mapsto a_{n}\right\}$ \\
\hline register map & $R$ & $::=$ & $\left\{v_{1} \mapsto r_{1}, \ldots, v_{n} \mapsto r_{n}\right\}$ \\
\hline type abbrev. map & $T$ & $::=$ & $\left\{t_{1} \mapsto \tau_{1}, \ldots, t_{n} \mapsto \tau_{n}\right\}$ \\
\hline instr. sequence & $S$ & $::=$ & $\iota ; S \mid$ branch | jmp \\
\hline instructions & $\iota$ & $::=$ & add $v_{d}, v_{1}, v_{2} \mid \cdots(53$ more $)$ \\
\hline types & $\tau$ & $::=$ & $\alpha|\top| \perp\left|\operatorname{int}_{32}\right| \exists \alpha . \tau \mid \ldots$ \\
\hline
\end{tabular}

Figure 11: LTAL Syntax

LTAL clauses, along with their soundness proofs in higherorder logic; checks the soundness proofs; and then runs the LTAL checker, which is a syntax-directed computation in our subset Prolog.

Chen et al. [7] describe the LTAL and the compiler that produces it; Tan et al. [19] describe the semantic model of the LTAL. In this paper we focus on the aspects of the LTAL calculus that enable it to be type-checked by our tiny trusted checker.

Because a source-language programmer never sees the LTAL program, we can design the LTAL calculus to be checkable in our very restricted language. To use the checker's limited support for dynamic clauses, we have arranged the LTAL so that: All identifiers in LTAL are small integers. No variables have the same identifier. Program labels, local variables, and type abbreviations are represented by disjoint sets of integers. To make the LTAL type system entirely syntaxdirected, we use explicit coercions to guide the typing rules, instead of relying on subtyping which would require a search.

We use the simple and limited arithmetic provided by the checker: addition, multiplication, and truncating division on 32-bit natural numbers. Other operators are synthesized: for example, such as $A>B$ is $\operatorname{div} B A 0$, using truncating division.

\subsection{Syntax}

The syntax is illustrated in Figure 11. An LTAL program consists of various maps (including type abbreviation declarations, label map, and register map), a set of function declarations, and a start label. Function declaration $f[\vec{\alpha}]\left(m, v_{1}\right.$ : $\left.\tau_{1}, \ldots, v_{n}: \tau_{n}\right) S$ defines a function (basic block) with label $f$, type parameters $\vec{\alpha}$, formal parameters $v_{1}: \tau_{1}, \ldots, v_{n}: \tau_{n}$, and function body $S$ which is a sequence of LTAL instructions. The function label $f$ is assigned a code pointer type $\operatorname{codeptr}[\vec{\alpha}]\left(v_{1}: \tau_{1}, \ldots, v_{n}: \tau_{n}\right)$.

The label environment $L$ is a map from program labels to their addresses. The register-allocation environment $R$ maps variables to temporaries (registers or spill locations). The type abbreviation environment $T$ maps type abbreviations to their expansions. Type abbreviations are used to gain concise type expressions and the type checker opens a type abbreviation only when needed. 


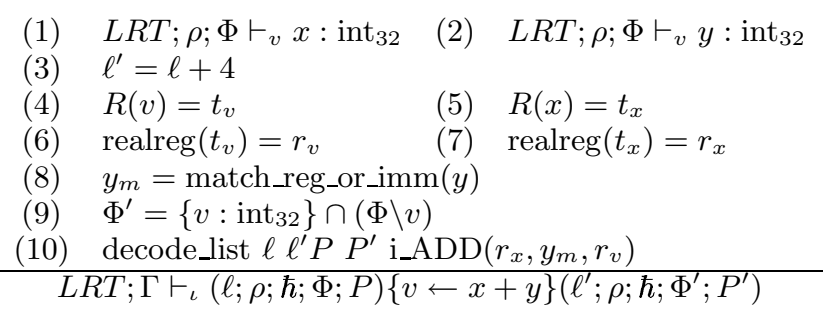

Figure 12: The typing rule of add instruction.

\subsection{Typing rules}

The LTAL has hundreds of clauses. Here we will show just one: a rule for Sparc add instruction, shown in Figure 12.

The first and second premises state that both $x$ and $y$ have type int $_{32}$, 32-bit integer type. Environment $L R T$ is label, register allocation, and type abbreviation maps. Address $\ell$ is the location of current instruction $v \leftarrow x+y$; address $\ell^{\prime}$ is the location of the next instruction. Premise (3) specifies that this instruction is 4 bytes long.

Premises (4), (5), (6), and (7) map variables to "temporaries" and temporaries to registers. They use the $R$ component of the $L R T$ environment; $R$ is a context managed with dynamic clauses.

Premise (8) matches a particular Sparc addressing mode; premise (9) relates the value typing contexts $\Phi, \Phi^{\prime}$ before and after execution of the current instruction. The $\Phi$ context is small (it just maps currently live local variables) and is represented as a list, not with dynamic atomic clauses.

The decode_list relation in premise (10) maps an instruction encoding (i.e., an integer) to its semantics. Specifically, it says that the instruction word at the beginning of machine code $P$ with length $\ell^{\prime}-\ell$ is an add instruction i_ADD $\left(r_{x}, y_{m}, r_{v}\right)$. Machine code $P$ is a sequence of integers (instruction words); the pair $\left(P, P^{\prime}\right)$ is a conventional Prolog difference-list.

The conclusion is like a Hoare-logic judgement. In environment $L R T$, the instruction $v \leftarrow x+y$ is at location $\ell$; the length of the instruction is $\ell^{\prime}-\ell$; this instruction does not affect type contexts $\rho$ or heap allocation environment $\hbar$; value context $\Phi$ becomes $\Phi^{\prime}$ after execution; the machine code at location $\ell^{\prime}$ is $P^{\prime}$.

For a real-life program, the generated maps $L, R$, and $T$ are very large: the sizes of $L$ and $R$ are approximately linear in the size of the program, and we intend to be able to typecheck programs with millions of instructions. In this typing rule, premises (4) and (5) look up the temporaries of $v$ and $x$ in map $R$; premise (8) looks up the temporary of $y$ if it is not an immediate. Therefore, an efficient environment management scheme really is necessary.

Such typing rules, though bigger and more complicated than the rules we presented for the even-odd system, can be executed by our simple subset Prolog interpreter.

\section{EXPERIMENTAL RESULTS}

We have measured our trusted checker on the even-odd microbenchmark (we expect to have measurements of the fullscale LTAL in the final version of this paper). Gross statistics about these proof schemes are as follows:

\begin{tabular}{|c|rrl}
\multicolumn{2}{c}{ EvenOdd } & LTAL & \\
\cline { 1 - 1 } Core Axioms & 341 & 341 & lines of LF \\
App-specific & 10 & 1522 & lines of LF \\
\cline { 1 - 1 } Expr. Ops. & 40 & 2 & lines of LF \\
\hline Sem. Model & 218 & $\sim 100,000$ & lines of LF \\
\cline { 1 - 1 } Hint Ops. & 10 & 500 & lines of LF \\
\cline { 1 - 1 } Clauses & 12 & 3,500 & lines of LF \\
\hline Expression & $7 N$ & $2 N$ & tokens \\
\cline { 1 - 1 } Hint & $4 N$ & $30 N$ & tokens \\
\hline
\end{tabular}

Lines of LF does not include blank lines and comments. Expression sizes for EvenOdd are measured with $N$ as the number of declarations, each declaration of the form let $x_{i}=$ $x_{j}+x_{k}$; which is 7 tokens per declaration. Expression sizes for LTAL are measured with $N$ as the number of machine instructions (32-bit integers) in the program to be proved safe, with two tokens per integer, for example:

2551193600 next_word 2181292040 next_word 2214748172 end

From this it should be clear why LTAL has only two Expression Operators; everything shown in Figure 11 is actually Hint Operators.

The logic program is the set of LTAL typing rules. There are several hundred LTAL clauses or typing rules, some of which take dozens of lines to write down, such as the one we showed in Section 6.2 for Sparc add instruction. The LTAL semantic model, which provides proofs of all these clauses, is rather intricate and is the subject of several other papers $[4,5,1,19]$.

Since the clauses are written in a subset of Prolog, we can execute them in a standard Prolog system. For each benchmark, we compare execution time in the (highly optimized) SICStus Prolog compiler with execution time in the (tiny) Flit interpreter.

\begin{tabular}{l|rr|rr|} 
& \multicolumn{2}{|c|}{ EvenOdd } & \multicolumn{2}{c|}{ LTAL } \\
Stage & SICS & Flit & SICS & Flit \\
\hline 1 (load axioms) & $\mathrm{n} / \mathrm{a}$ & 0.01 & $\mathrm{n} / \mathrm{a}$ & $?$ \\
2 (check scheme) & $\mathrm{n} / \mathrm{a}$ & 0.07 & $\mathrm{n} / \mathrm{a}$ & $?$ \\
\hline 3 (run scheme) & & & & \\
$N=100$ & 0.002 & 0.01 & $\mathrm{n} / \mathrm{a}$ & $\mathrm{n} / \mathrm{a}$ \\
$N=1000$ & 0.030 & 0.79 & $\mathrm{n} / \mathrm{a}$ & $\mathrm{n} / \mathrm{a}$ \\
$N=10000$ & 1.460 & 61.90 & $\mathrm{n} / \mathrm{a}$ & $\mathrm{n} / \mathrm{a}$ \\
\hline$N=870$ & $\mathrm{n} / \mathrm{a}$ & $\mathrm{n} / \mathrm{a}$ & 0.106 & $?$ \\
$N=1816$ & $\mathrm{n} / \mathrm{a}$ & n/a & 0.206 & $?$ \\
\hline
\end{tabular}

All times are in seconds on a $2.2 \mathrm{GHz}$ Pentium IV. The system parses axioms and proof-check clauses once, and then it is prepared to accept arbitrary expressions (with hints) and to execute clauses to check safety. Flit is slower than SICStus Prolog, but its speed is still adequate for many kinds of applications. For LTAL, Flit can be expected by be slower by a larger factor, because clause-search takes time linear in the number of static clauses, and the LTAL proof- 
scheme has many more clauses.

Of course, execution in SICStus loses the benefits of the tiny trusted base: in that mode we don't mechanically connect the soundness proof for the LTAL clauses to the actual SICStus execution, and the SICStus Prolog compiler and interpreter also become part of the trusted base.

The Flit software itself now comprises about 1100 lines of C code: about 800 as described in Section 1 for parsing the axioms, reading proof graphs, and LF checking the proofs, about 200 for the logic-program interpreter, and about 100 to manage the stages described in Section 5.1.

Necula's oracle-based Prolog interpreter [12] is about 800 lines of $\mathrm{C}$ code. It should be straightforward to use our style of LF proof-checking of Prolog clauses, but use oracle-based execution instead of our interpreter. Then, instead of an 1100-line C program, we would have an 1700-line program. In such a system, the proof witnesses would be just as tiny as Necula's, and the trusted base would be somewhat larger than that of the system we have described in this paper.

\section{CONCLUSION}

To make a trustworthy proof-checker with small witnesses, one should define a language for proof-schemes, with a way to represent and check soundness theorems for the proof schemes; then one should implement an interpreter to execute the proof scheme on the theorem and the witness.

Pollack explained much of this in "How to believe a machinechecked proof" [17], as follows:

... I suggest that the "programming language" for the checking program be a logical framework [such as] the Edinburgh Logical Framework .... we [could] program a checker in the internal language of the framework .... The question then arises: where will we find a believable implementation of a logical framework?

We ask you to believe very little. Our implementation is based on LF, higher-order logic, and a small subset of pure Prolog, all of which are well understood; and our implementation is about as small as possible - that is, to trust our system there are only 1100 lines of code that you have to understand.

\section{REFERENCES}

[1] Amal J. Ahmed, Andrew W. Appel, and Roberto Virga. A stratified semantics of general references embeddable in higher-order logic. In In Proceedings of the 17th Annual IEEE Symposium on Logic in Computer Science (LICS 2002), July 2002.

[2] Andrew W. Appel. Foundational proof-carrying code. In Symposium on Logic in Computer Science (LICS '01), pages 247-258. IEEE, 2001.

[3] Andrew W. Appel and Edward W. Felten. Proof-carrying authentication. In 6th $A C M$ Conference on Computer and Communications Security. ACM Press, November 1999.
[4] Andrew W. Appel and Amy P. Felty. A semantic model of types and machine instructions for proof-carrying code. In POPL '00: The 27th ACM SIGPLAN-SIGACT Symposium on Principles of Programming Languages, pages 243-253, New York, January 2000. ACM Press.

[5] Andrew W. Appel and David McAllester. An indexed model of recursive types for foundational proofcarrying code. ACM Trans. on Programming Languages and Systems, 23(5):657-683, Sept. 2001.

[6] Andrew W. Appel, Neophytos Michael, Aaron Stump, and Roberto Virga. A trustworthy proof checker. In Iliano Cervesato, editor, Foundations of Computer Security workshop, pages 37-48. DIKU, July 2002.

[7] Juan Chen, Dinghao Wu, Andrew W. Appel, and Hai Fang. A provably sound TAL for back-end optimization. In PLDI '03: Proceedings of the 2003 ACM SIGPLAN Conference on Programming Language Design and Implementation, New York, June 2003. ACM Press.

[8] Robert Harper, Furio Honsell, and Gordon Plotkin. A framework for defining logics. Journal of the ACM, 40(1):143-184, January 1993.

[9] Christopher League, Zhong Shao, and Valery Trifonov. Precision in practice: A type-preserving Java compiler. In 12th International Conference on Compiler Construction (CC'03), page to appear, April 2003.

[10] George Necula. Proof-carrying code. In 24th ACM SIGPLAN-SIGACT Symposium on Principles of Programming Languages, pages 106-119, New York, January 1997. ACM Press.

[11] George C. Necula and Peter Lee. Efficient representation and validation of proofs. In In Proceedings of the 13th Annual Symposium on Logic in Computer Science, 1998.

[12] George C. Necula and S. P. Rahul. Oracle-based checking of untrusted software. In POPL 2001: The 28th ACM SIGPLAN-SIGACT Symposium on Principles of Programming Languages, pages 142-154. ACM Press, January 2001.

[13] Frank Pfenning. Logic programming in the LF logical framework. In Gérard Huet and Gordon Plotkin, editors, Logical Frameworks, pages 149-181. Cambridge University Press, 1991.

[14] Frank Pfenning. Elf: A meta-language for deductive systems. In A. Bundy, editor, Proceedings of the 12th International Conference on Automated Deduction, pages 811-815, Nancy, France, June 1994. Springer-Verlag LNAI 814.

[15] Frank Pfenning and Carsten Schürmann. System description: Twelf - a meta-logical framework for deductive systems. In H. Ganzinger, editor, Proceedings of the 16th International Conference on Automated Deduction (CADE-16), pages 202-206, Trento, Italy, July 1999. Springer-Verlag. LNAI 1632. 
[16] Frank Pfenning and Carsten Schürmann. Twelf User's Guide (Version 1.4). Carnegie-Mellon Univ., 2002.

[17] Robert Pollack. How to believe a machine-checked proof. In G. Sambin and J. Smith, editors, Twenty Five Years of Constructive Type Theory. oup, 1998.

[18] David A. Schmidt. Denotational Semantics: A Methodology for Language Development. Allyn and Bacon, Boston, 1986.

[19] Gang Tan, Kedar Swadi, Dinghao Wu, and Andrew W. Appel. Construction of a semantic model for a typed assembly language. March 2003.

\section{APPENDIX}

\section{A. MACHINE CHECKABLE PROOFS}

To illustrate the format of the machine-checked soundness proofs of the type-checking clauses, here we will show the proofs related to the rule BindTy.

Since the proof is written in LF, we begin with a brielf introduction to LF. LF is based on the $\lambda$-calculus with dependent types, and it has syntactic entities at three levels: objects, types, and kinds. Types classify objects and kinds classify families of types. A deductive system is represented in LF using the judgements-as-types and derivationsas-terms principle [8]: judgements (theorems) are represented as types, and derivations (proofs) are represented as terms whose type is the representation of the judgement (theorem) that they prove. In this way proof checking of the object logic is reduced to type checking of the LF terms.

In general, a definition in Twelf (an implementation of LF with many extended features) has the form: name $: \tau=\exp$. including the dot. The type $\tau$ encodes the theorem to be proved, and exp is a term of type $\tau$. By judgements-as-types and derivations-as-terms principle, term exp is a proof of the theorem that $\tau$ encodes. And the name stands for the whole term exp with type $\tau$, i.e. the theorem and the proof. $\mathrm{LF}$ and Twelf also permit introducing constructors with the form name $: \tau$. In our case, we have:

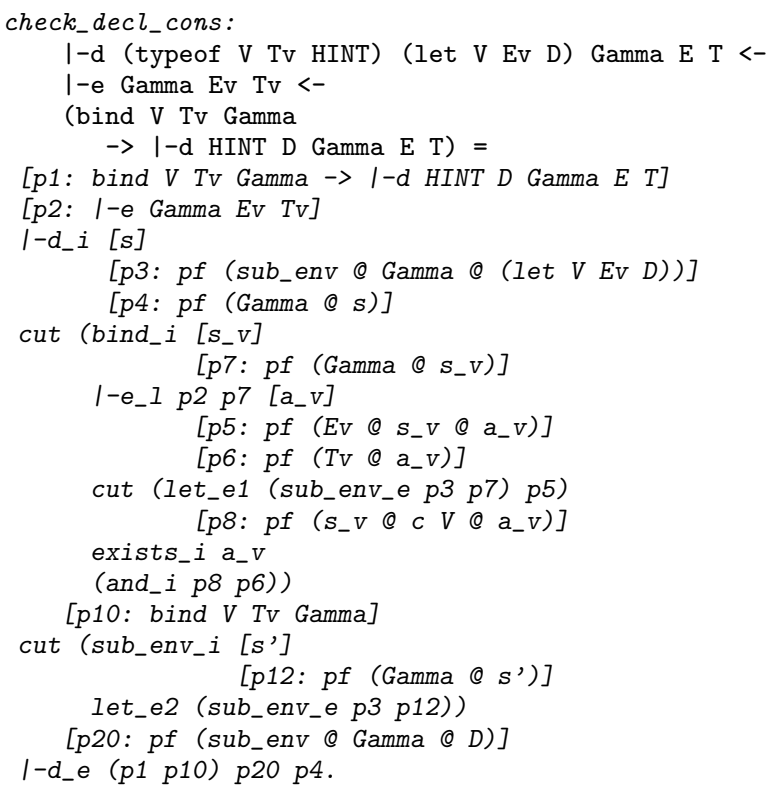

The name of this theorem is check_decl_cons (in Section 3, we used BindTy for presentation purpose). The theorem itself is the type between : and =; and the proof is the term after $=$.

A logic programming clause in Twelf of the form:

$$
f x y A \leftarrow g y \leftarrow h x A .
$$

corresponds to the following Prolog clause:

$$
f(x, y, A):-g(y), h(x, A) .
$$

So apprarently the type above between : and = encodes the BindTy rule with the forward arrow $\rightarrow$ interpreted as dynamic clauses as we discussed in Section 3.1.

The notation " $[\mathrm{x}: \mathrm{t}] \mathrm{A}$ " denotes $\lambda \mathrm{x}: \mathrm{t} . \mathrm{A}$. The proof above we first introduce two $\lambda$-bindings; that is, we assume that the two premises of the typing rule hold. Then we use the $1-d$ introduction rule $1-d_{-} i$ to get a proof of

I-d (typeof V Tv HINT) (let V Ev D) Gamma E T, i.e. the conclusion.

The rule $1-d_{-} i$ introduces three $\lambda$-bindings: $s, p 3$, and $p 4$. Note that the type of $s$ is omitted and Twelf will reconstruct it to a State type. Lemma cut is as follows:

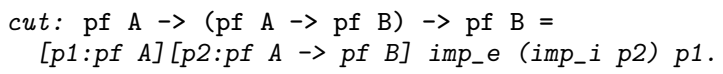

The imp_i and imp_e (modus ponens) are introduction and elimination lemmas for implication. In general, it means if we have a proof of $A$, and a function which maps a proof of $A$ to a proof of $B$, then we can get a proof of $B$. This is very similar to imp_e or modus ponens, but cut use LF function type $\rightarrow$ instead of object implication. When using cut, we first prove some formula $A$, then bind this proof (give it a name so that we can refer to it later) and continues to prove the goal ( $B$ in this case). The @ is the object logic level term application.

In this way, it should be quite straightforward to follow the proofs above. Thus the description of the remaining proofs is omitted. 